\title{
Fibre Area Correction Factors (FACF) for the extended rules-of-mixtures for natural fibre reinforced composites
}

\author{
John Summerscales ${ }^{\mathrm{a}^{*}}$, Amandeep Singh Virk ${ }^{\mathrm{b}}$ and Wayne Hall ${ }^{\mathrm{c}}$ \\ ${ }^{a}$ Composites Engineering/Materials and Structures Research Group (CE/MAST), \\ School of Engineering, Computing and Mathematics (SECaM), Faculty of Science and Engineering, \\ University of Plymouth, Drake Circus, Plymouth, PL4 8AA, United Kingdom. \\ ${ }^{b}$ Stantec, 10160-112 Street, Edmonton, Alberta T5K 2L6, Canada. \\ ${ }^{c}$ Mechanical Engineering G09 1.51, Griffith University, Gold Coast campus, Parklands Dr, Southport, Qld 4215, Australia.
}

\begin{abstract}
The prediction of the properties of fibre-reinforced composite materials normally uses rules-of-mixture (RoM), but accuracy is constrained by the validity of data input to the model. A common error is the use of the fibre "apparent diameter" to determine the cross-sectional area of the polygonal fibre, and hence the fibre modulus and strength values are low. New equations are presented which can produce improved predictions of the in-plane moduli and strengths of natural fibre composites. Provisional data for the Fibre Area Correction Factors required by the new equations, based on recent publications in the scientific literature, are provided. [copyright information to be updated in production process]
\end{abstract}

Keywords: composite; fibre area correction factor; natural fibre; rules-of-mixtures; vegetal fibres

\section{Introduction}

The mechanical properties of fibre-reinforced composites may be predicted using the rules-of-mixture (RoM) with results dependent on the quality of the input data. It is common practice to determine the moduli and strengths of fibres using "standard" test methods (e.g. Grafil test 101.13) where the fibre diameter measurement (Grafil test 102.13) is determined by optical microscopy using a continuous length of each filament mounted on a test card for tensile testing. This approach is sensible for synthetic fibres with circular cross-section, but (both technical and ultimate) natural fibres normally have polygonal cross-section.

To avoid errors through the assumption of circular cross-section, either of two alternative approaches may be adopted. Automated non-contact dimensional measurement of fibres is available [1] where the test fibre is rotated in a laser scanning micrometer to generate a more accurate measurement but the system cannot see concave features. Alternatively, the effective cross-sectional area can be determined by weighing the fibre and calculating cross-sectional area as mass/(density*length). However, the determination of mass requires accurate measurement of a small sample, the density of the fibre may vary with local composition, and the mean cross-sectional area may not be an accurate figure for the specific sample length under test. Further, the measurement of density for hydrophilic fibres is not trivial. For example, jute is quoted as having a density of $1300-1460 \mathrm{~kg} \cdot \mathrm{m}^{-3}$ [2]. However, one study using Archimedes principle in combination with vacuum degassing of the sample reported figures for jute fibres of $1669 \pm 37$ $\mathrm{kg} \cdot \mathrm{m}^{-3}$ in water/Ilfotol or $1652 \pm 37 \mathrm{~kg} \cdot \mathrm{m}^{-3}$ in acetone [3]. Drying in air and reweighing (removal of $\sim 4 \%$ moisture) resulted in a density of $1570 \pm 43 \mathrm{~kg} \cdot \mathrm{m}^{-3}$ which is comparable with the highest values quoted in the literature for natural fibres. 
Virk et al [4] have extended RoM for the estimation of in-plane composite properties by introducing a fibre area correction factor (FACF) to account for the common practice of assuming a circular cross-section for natural fibres when the true cross-section is polygonal. The FACF is calculated as the mean circular cross-sectional area (CSA), derived from the apparent diameter, divided by the mean true CSA for a statistically significant number of the fibres. The proposed enhancement of RoM improves the physical assumptions in the mathematical derivation of the model by correcting the cross-sectional area used in the calculation. The over-estimated area, when assuming circular CSA, results in low fibre moduli and strengths. As FACF > 1, this increases the calculated mechanical properties.

\subsection{Equations}

The extended rules-of-mixture [4] for the in-plane modulus of composites in general (Equation 1), and for strength (only for quasi-unidirectional composites) by extension of the Kelly-Tyson equation (Equation 2), are:

$$
\begin{array}{lr}
E_{\mathrm{c}}=\kappa \eta_{\mathrm{d}} \eta_{1} \eta_{\mathrm{o}} V_{\mathrm{f}} E_{\mathrm{f}}+\mathrm{V}_{\mathrm{m}} E_{\mathrm{m}} & \text { Equation 1 } \\
\sigma_{\mathrm{c}}{ }^{\prime}=\kappa V_{\mathrm{f}} \sigma_{\mathrm{f}}{ }^{\prime}+V_{\mathrm{m}} \sigma_{\mathrm{m}^{*}} & \text { Equation 2 }
\end{array}
$$

where $E_{x}$ is elastic modulus, $V_{x}$ is component volume fraction, $\kappa$ is the FACF, $\eta_{d}$ is the fibre diameter distribution factor, $\eta_{1}$ is the fibre length distribution factor, $\eta_{\mathrm{o}}$ is the fibre orientation distribution factor, $\sigma_{\mathrm{x}}{ }^{\prime}$ is the material strength (assuming circular fibre CSA), and $\sigma_{\mathrm{m}^{*}}$ is the stress in the matrix at the failure strain of the fibre. Subscripts $c, f$ and $m$ denote composite, fibre and matrix respectively.

Equation 1 has a number of underlying assumptions, compiled from [5-8]:

- the fibres are continuous with uniform cross-section and lie parallel to each other.

- the fibres are homogeneous and linear elastic

$\circ \quad$ if the fibres are orthotropic, then a more general form of the equation may be required.

- the matrix is homogeneous and linear elastic

- both the fibre and the matrix are free of voids

- the interface is completely bonded with "no slip" between adjacent layers

- there is no transition region (no third party material) between the matrix and the reinforcement

- all displacements and strains are small

- there is equal strain in the fibre and the matrix

- each composite ply is orthotropic, macroscopically homogeneous and linearly elastic

- each lamina has uniform thickness across the layer

- the transverse shear stresses on the upper and lower surfaces are zero [9]

- the laminate deforms in accordance with the Kirchoff-Love plate theory:

- all normals to the mid-surface remain straight and normal after deformation

$\circ$ the thickness is unchanged after deformation

- Poisson's strains are neglected (the Poisson's ratio of the fibre and the matrix are the same)

$\circ \quad$ if the Poisson's ratios differ, then the neglected term has been shown to be of the order of the square of the difference between the two values [5]

\section{Validation}

For the jute fibres in Virk's doctoral studies [10], a FACF of 1.42 was obtained. For the Virk composites, the experimental moduli were 8.47 $\pm 1.18 \mathrm{GPa}$ (undyed fibre/unpigmented resin) or 8.19 $\pm 0.6 \mathrm{GPa}$ (dyed/pigmented) respectively. The moduli predicted using Equation 1 with both $\kappa$ and $\eta_{\mathrm{d}}$ at unity (classical equation) was $6.44 \mathrm{GPa}$ and with FACF of 1.42 was $\mathbf{8 . 2 4} \mathrm{GPa}$. The elastic moduli calculated for jute fibre composites, with data from a number of literature sources, clearly indicate that the FACF prediction is closer to the experimental values in most cases [4]. 
The experimental strengths of the Virk jute composites were 101.0 \pm 17.2 (undyed / unpigmented) and 100.0 \pm 5.7 MPa (dyed/pigmented) respectively. The predicted strengths were calculated using the standard Kelly-Tyson equation (Equation 2 with $\kappa=1$ ) and using Equation 2 above. In each case, the predicted fibre strength differed dependent on whether a multiple data set (MDS) weak link scaling (WLS) model or a natural logarithm interpolation model (NLIM) statistical model was used in the analysis of the fibre data. The standard Kelly-Tyson equation predicted 79.2 MPa (NLIM) and 73.1 MPa (MDS), while the FACF corrected values were 102.9 MPa (NLIM) and 95.0 MPa (MDS) respectively. The experimental mean values for composite strength lie between the two Equation 2 predictions.

\section{Fibre Area Correction Factors for other systems}

Terasaki et al [11], calculated the cross-sectional area of natural fibers by measuring their projection widths and assumed an elliptical rather than circular cross-section to calculate the cross-sectional area. Nitta et al [12], from the same group, used a new data-based approximation (DBA) method, which is a statistical distribution of actual fibre cross-sectional area obtained from many cross-sectional pictorial images, to obtain the same FACF.

Aslan et al [13] investigated the variability in tensile properties of flax fibres and found that the inaccuracy in the determination of the cross sectional area of the fibres was one major reason for the variability in calculated fibre properties.

Thomason et al [14] studied CSA and variability for flax or sisal fibres and concluded "that fibre diameter measurement is not an attractive method for accurate estimation of cross-sectional area of these natural fibres".

Soatthiyanon et al $[15,16]$ studied the tensile properties of 113 flax technical fibres, using an experimentally determined FACF of 2.70 to account for the non-circularity of the fibres. Taking this into account for the single fibre tests, the back-calculated modulus of the flax fibres was $52.4 \mathrm{GPa}$, within $6 \%$ of that obtained from the single fibre tests, while the strength was $936 \mathrm{MPa}$ within $7 \%$.

Garat et al [17] have recently published data comparing the apparent and true CSA for five fibres (flax, hemp, nettle, palm and sisal). Assuming that fibre mounted on test-cards settle with their centre of gravity at the lowest point, the measured diameter will be the maximum value.

Ferrante et al [18] calculated FACF for banana peel fibres based on true total CSA and taking into account the luminal cavity. The hollow fibre will have a lower density than a fibre without lumen, but the intra-fibre void may not be detrimental to the performance of a natural fibre composite under axial loading [19]. Baley et al [20] have suggested that the lumen may encourage crack initiation in transverse loading.

Table 1 and Figure 1 present provisional FACF values for fibres using data from the open literature (arithmetic average used for range of diameter data), plus values obtained by Plymouth students. The reported FACF appear to be for technical (apparent diameter $>\sim 25 \mu \mathrm{m}$ ), not ultimate, fibres with the exception of two data points for flax.

This initial data may permit more accurate determination of fibre, and hence calculation of composite, properties. More research is required to establish the degree of dependence of FACF values on the fibre batch under consideration, and whether there are systematic changes in FACF with increasing fibre average diameter or the fibre processing undertaken. If that is not the case, it may be necessary to determine FACF for the specific batch of fibre used.

\section{Summary}

New equations have been presented which may improve predictions of the in-plane moduli and strengths of natural fibre composites. Provisional data for the Fibre Area Correction Factors required by the new equations, based on recent data in the literature, are provided. More research is required to establish if the FACF values are dependent on the fibre batch under consideration, and on whether the FACF changes with increasing fibre average diameter. 
Table 1. Fibre Area Correction Factors $(F A C F)$ for natural fibres $(B=$ bast, $F=$ fruit, $G=$ grass, $L=$ leaf $)$ $(*$ format $=$ median $($ minimum - maximum $))$.

\begin{tabular}{|c|c|c|c|c|c|}
\hline Plant species & & Apparent diameter $(\mu \mathrm{m})$ & True CSA $\left(\mu \mathrm{m}^{2}\right)$ & FACF & Reference \\
\hline Palm & $\mathrm{L}$ & $145(120-263)^{*}$ & $17610(11147-54010)^{*}$ & 0.94 & Garat et al [17]. \\
\hline Flax & $\mathrm{B}$ & $91(49-140)^{*}$ & $6148(1929-11030)^{*}$ & 1.06 & Garat et al [17]. \\
\hline Sisal & $\mathrm{L}$ & $163(113-263)^{*}$ & $18845(7954-49530)^{*}$ & 1.11 & Garat et al [17]. \\
\hline Flax & $\mathrm{B}$ & $17.3 \pm 2.1$ & & 1.12 & Brierley [21]. \\
\hline Nettle & $\mathrm{B}$ & $90(56-161)^{*}$ & $5199(1449-14170)^{*}$ & 1.22 & Garat et al [17]. \\
\hline Curaua & $\mathrm{L}$ & 92.3 & & 1.27 & Terasaki et al [11]. \\
\hline Maize & $\mathrm{B}$ & 221 & 29472 & 1.30 & Connolly [22] \\
\hline Hemp & $\mathrm{B}$ & $88(47-173)^{*}$ & $4411(1605-16990)^{*}$ & 1.38 & Garat et al [17]. \\
\hline Flax & $\mathrm{B}$ & $20.4 \pm 9.9$ & $236 \pm 115$ & 1.39 & Aslan et al [13] \\
\hline Jute & $\mathrm{B}$ & 58.6 & & 1.42 & Virk et al [10]. \\
\hline Kenaf & $\mathrm{B}$ & 96.8 & 5010 & 1.47 & Nitta et al [12] \\
\hline Kenaf & $\mathrm{B}$ & 96.8 & & 1.47 & Terasaki et al [11]. \\
\hline Bamboo & G & 136.1 & & 1.60 & Terasaki et al [11]. \\
\hline Sisal & $\mathrm{L}$ & $162-359$ & & 1.99 & Thomason et al [14]. \\
\hline Banana & $\mathrm{F}$ & $131 \pm 22$ & $5429 \pm 1366$ (includes lumen) & 2.54 & Ferrante at al [18] \\
\hline Flax & $\mathrm{B}$ & $109-218$ & & 2.55 & Thomason et al [14]. \\
\hline Flax & $\mathrm{B}$ & $82 \pm 21$ & & 2.70 & Soatthiyanon et al $[15,16]$. \\
\hline Banana & $\mathrm{F}$ & $131 \pm 22$ & $4624 \pm 1210$ (excludes lumen) & 2.98 & Ferrante at al [18] \\
\hline
\end{tabular}

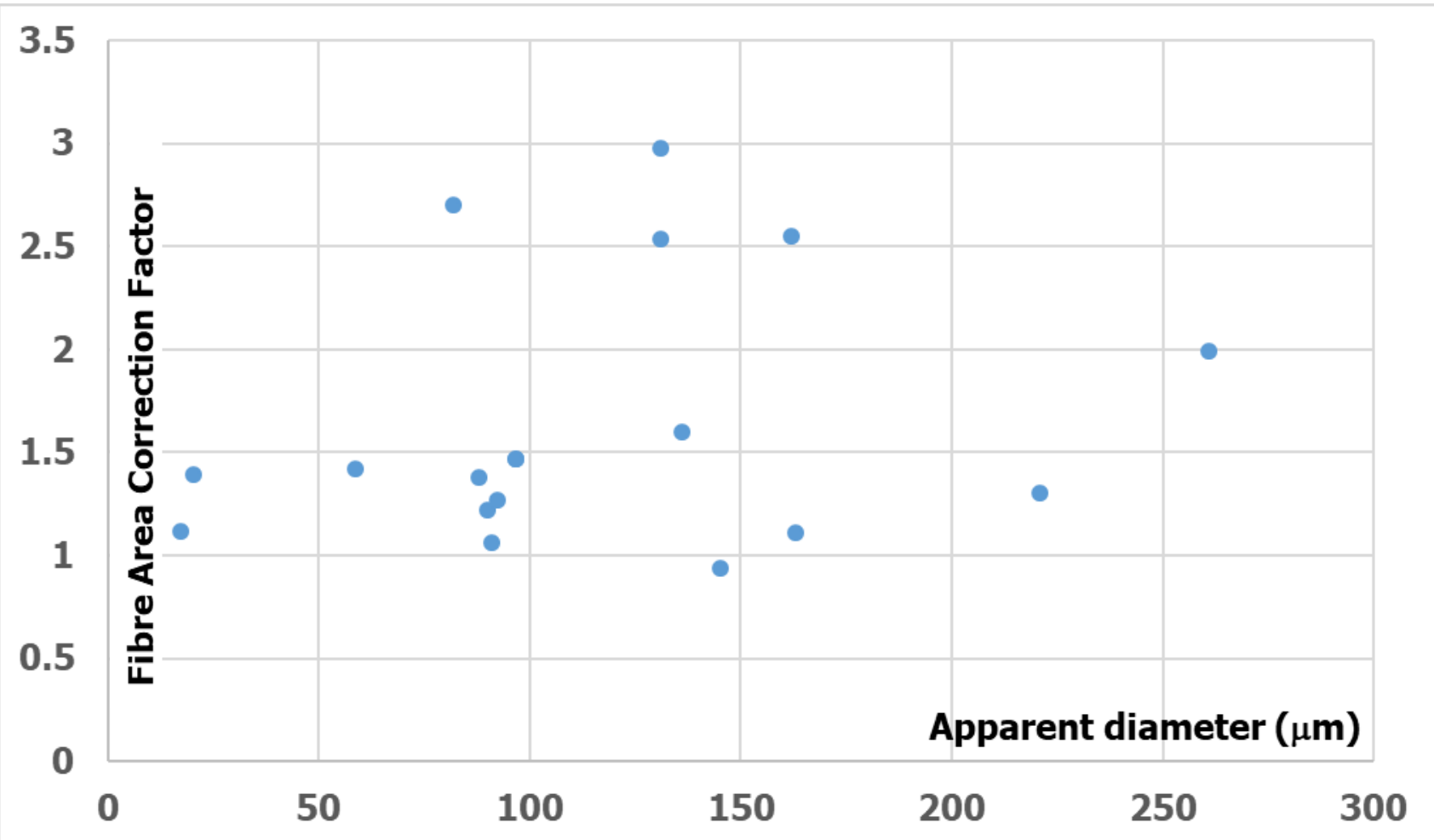

Fig. 1: Fibre area correction factor plotted against apparent diameter for the data in Table 1. 


\section{References}

[1] Dia-stron, https://www.diastron.com/testing-solutions/dimensional/, accessed 09 January 2020.

[2] H Singh, JIP Singh, S Sehijpal, V Dhawan and SK Tiwari, A brief review of jute fibre and its composites, Materials Today: Proceedings, 5(2018) 28427-28437.

[3] R.K. Cullen, M.M. Singh and J. Summerscales, Characterisation of natural fibre reinforcements and composites, Journal of Composites, 2013, article ID 416501 (https://doi.org/10.1155/2013/416501)

[4] A.S. Virk, W. Hall, J. Summerscales, Modulus and strength prediction for natural fibre composites, Materials Science and Technology, 28 (2012) 864-871.

[5] A. Brent Strong, Fundamentals of Composites Manufacturing: materials, methods and applications, Society for Manufacturing Engineers, Dearbon MI, 1989. ISBN 0-87263-358-6.

[6] R.F. Gibson, Principles of Composite Material Mechanics, McGraw-Hill, 1994. ISBN 0-07-023451-5. Third edition, 2012. ISBN 978-1-4398-5005-3.

[7] J.N. Reddy, Mechanics of laminated plates and shells: theory and analysis - second edition, CRC Press, Boca Raton FL, 2003. ISBN 978-0-84931-592-3.

[8] P.M. Mohite, Introduction to classical plate theory, National Programme on Technology Enhanced Learning India, 2012, accessed 21 December 2016.

[9] C Basdogan, Transverse Shear Stresses in Beams, MDesign course notes, Koç University, no date, accessed 21 December 2016.

[10] A.S. Virk, Numerical models for natural fibre composites with stochastic properties, $\mathrm{PhD}$, University of Plymouth, 2010. (http://hdl.handle.net/10026.1/517).

[11] Y. Terasaki, J. Noda and K. Goda, Strength evaluation of green composite with variation in cross-sectional area of plant-based natural fibers, Advanced Materials Research, 79-82 (2009) 235-238 [Special issue on Multi-Functional Materials and Structures II edited by Y. Yin and X. Wang].

[12] Y. Nitta, K. Goda, J.Noda, W.-I. Lee, Cross-sectional area evaluation and tensile properties of alkali-treated kenaf fibres, Composites Part A: Applied Science and Manufacturing, 49 (2013) 132-138.

[13] M. Aslan, G. Chinga-Carrasco, B.F. Sørensen, B Madsen, Strength variability of single flax fibres, Journal of Materials Science, 46 (2011) 6344-6354.

[14] J.L. Thomason, J. Carruthers, J. Kelly, G. Johnson, Fibre cross-section determination and variability in sisal and flax and its effects on fibre performance characterisation, Composites Science and Technology, 71 (2011) $1008-1015$.

[15] N. Soatthiyanon, Separation and characterisation of elementary kenaf fibres as reinforcement in high-density polyethylene-matrix composites and tensile behaviour of flax fibres as reinforcement in vinyl ester-matrix composites, PhD thesis, University of New South Wales, 2014 (http://handle.unsw.edu.au/1959.4/53585).

[16] N. Soatthiyanon, A. Crosky and M.T. Heitzmann, Comparison of experimental and calculated tensile properties of flax fibres, In D. Fernando, J.-G. Teng, J.L. Torero (editors), Proceedings of the Second International Conference on Performance-based and Life-cycle Structural Engineering (PLSE 2015), Brisbane QLD, Australia, 9-11 December 2015, 116-120.

[17] W. Garat, S. Corn, N. Le Moigne, J. Beaugrand, A. Bergeret, Analysis of the morphometric variations in natural fibres by automated laser scanning: towards an efficient and reliable assessment of the cross-sectional area, Composites Part A: Applied Science \& Manufacturing, 108 (2018) 114-123.

[18] A. Ferrante, C. Santulli, J. Summerscales. Evaluation of Tensile Strength of Fibers Extracted from Banana Peels. Journal of Natural Fibers, accepted/not yet assigned to an issue. DOI: 10.1080/15440478.2019.1582000

[19] D.U. Shah, P.J. Schubel, M.J. Clifford, P. Licence, Mechanical property characterization of aligned plant yarn reinforced thermoset matrix composites manufactured via vacuum infusion, Polymer-Plastics Technology and Engineering, 53 (2014) 239-253.

[20] C. Baley, Y. Perrot, F. Busnel, H. Guezenoc, P Davies, Transverse tensile behaviour of unidirectional plies reinforced with flax fibers, Mater. Lett., 60 (2006) 2984-2987.

[21] M.J. Brierley, Fibre area correction factor (FACF) for flax fibre reinforcements, BEng (honours) Mechanical Engineering with Composites project report, University of Plymouth, April 2014.

[22] S. Connolly, Agricultural crop residue as a reinforcement in composite material, BEng (honours) Mechanical Engineering with Composites project report, University of Plymouth, May 2019. 